\title{
ELLIPTIC WEYL GROUP ELEMENTS AND UNIPOTENT ISOMETRIES WITH $p=2$
}

\author{
GEORGE LUSZTIG AND TING XUE
}

\begin{abstract}
Let $G$ be a classical group over an algebraically closed field of characteristic 2 and let $C$ be an elliptic conjugacy class in the Weyl group. In a previous paper the first named author associated to $C$ a unipotent conjugacy class $\Phi(C)$ of $G$. In this paper we show that $\Phi(C)$ can be characterized in terms of the closure relations between unipotent classes. Previously, the analogous result was known in odd characteristic and for exceptional groups in any characteristic.
\end{abstract}

\section{INTRODUCTION}

0.1. Let $G$ be a connected reductive algebraic group over an algebraically closed field $\mathbf{k}$ of characteristic $p \geq 0$. Let $\underline{\underline{G}}$ be the set of unipotent conjugacy classes in $G$. Let $\underline{\mathbf{W}}$ be the set of conjugacy classes in the Weyl group $\mathbf{W}$ of $G$. For $w \in \mathbf{W}$ and $\gamma \in \underline{\underline{G}}$ let $\mathfrak{B}_{w}^{\gamma}$ be the variety of all pairs $(g, B)$ where $g \in \gamma$ and $B$ is a Borel subgroup of $G$ such that $B$ and $g B g^{-1}$ are in relative position $w$. For $C \in \underline{\mathbf{W}}$ and $\gamma \in \underline{G}$ we write $C \dashv \gamma$ when for some (or equivalently any) element $w$ of minimal length in $C$ we have $\mathfrak{B}_{w}^{\gamma} \neq \emptyset$. In [L1, 4.5] a natural surjective map $\Phi: \underline{\mathbf{W}} \rightarrow \underline{\underline{G}}$ was defined. When $p$ is not a bad prime for $G$, the map $\Phi$ can be characterized in terms of the relation $C \dashv \gamma$ as follows (see [L1, 0.4]):

(a) If $C \in \underline{\mathbf{W}}$, then $\Phi(C)$ is the unique unipotent class of $G$ such that $C \dashv \Phi(C)$ and such that if $\gamma^{\prime} \in \underline{\underline{G}}$ satisfies $C \dashv \gamma^{\prime}$, then $\Phi(C)$ is contained in the closure of $\gamma^{\prime}$.

If $p$ is a bad prime for $G$, then the definition of the map $\Phi$ given in [L1] is less direct; one first defines $\Phi$ on elliptic conjugacy classes by making use of the analogous map in characteristic 0 and then one extends the map in a standard way to nonelliptic classes. It would be desirable to establish property (a) also in bad characteristic. To do this it is enough to establish (a) in the case where $C$ is elliptic (see the argument in [L1, 1.1].) One can also easily reduce the general case to the case where $G$ is almost simple; moreover, it is enough to consider a single $G$ in each isogeny class. The fact that (a) holds for $C$ elliptic with $G$ almost simple of exceptional type (with $p$ a bad prime) was pointed out in [L2, 4.8(a)]. It remains then to establish (a) for $C$ elliptic in the case where $G$ is a symplectic or a special orthogonal group and $p=2$. This is achieved in the present paper. In fact, Theorem 1.3 establishes (a) with $C$ elliptic in the case where $G$ is $S p_{2 n}(\mathbf{k})$ or $S O_{2 n}(\mathbf{k})(p=2)$; then (a) for $G=S O_{2 n+1}(\mathbf{k})(p=2)$ follows from the analogous

Received by the editors April 4, 2011 and, in revised form, November 3, 2011.

2010 Mathematics Subject Classification. Primary 20 G99.

The first author was supported in part by the National Science Foundation. 
result for $S p_{2 n}(\mathbf{k})$ using the exceptional isogeny $S O_{2 n+1}(\mathbf{k}) \rightarrow S p_{2 n}(\mathbf{k})$. Thus the results of this paper establish (a) for any $G$ without restriction on $p$.

0.2. If $w \in \mathbf{W}$ and $\gamma \in \underline{\underline{G}}$, then $G_{a d}$ (the adjoint group of $G$ ) acts on $\mathfrak{B}_{w}^{\gamma}$ by $x:(g, B) \mapsto\left(x g x^{-1}, x B x^{-\overline{1}}\right)$. Let $C \in \underline{\mathbf{W}}$ be elliptic. Let $\gamma=\Phi(C)$. The following result is proved in [L2, 0.2$]$.

(a) For any $w \in C$ of minimal length, $\mathfrak{B}_{w}^{\gamma}$ is a single $G_{a d}$-orbit.

The following converse of (a) appeared in [L2, 3.3(a)] in the case where $p$ is not a bad prime for $G$ and in the case where $G$ is almost simple of exceptional type and $p$ is a bad prime for $G$ (see also [L1, 5.8(c)]):

(b) Let $\gamma^{\prime} \in \underline{\underline{G}}$. If $C \dashv \gamma^{\prime}$ and $\gamma^{\prime} \neq \Phi(C)$, then for any $w \in C$ of minimal length, $\mathfrak{B}_{w}^{\gamma^{\prime}}$ is a union of infinitely many $G_{a d}$-orbits.

Using 0.1 (a) we see as in the proof of [L1, 5.8(b)] that (b) holds for any $G$ without restriction on $p$. Namely, from [L1, 5.7(ii)] we see that $\mathfrak{B}_{w}^{\gamma^{\prime}}$ has pure dimension equal to $\operatorname{dim} \gamma^{\prime}+l(w)$ where $l(w)$ is the length of $w$ and $\mathfrak{B}_{w}^{\gamma}$ has pure dimension equal to $\operatorname{dim} \gamma+l(w)$. Also, by [L1, 5.2], the action of $G_{a d}$ on $\mathfrak{B}_{w}^{\gamma^{\prime}}$ or $\mathfrak{B}_{w}^{\gamma}$ has finite isotropy groups. Thus, $\operatorname{dim} \mathfrak{B}_{w}^{\gamma}=\operatorname{dim} G_{a d}$ (see (a)) and to prove (b) it is enough to show that $\operatorname{dim} \mathfrak{B}_{w}^{\gamma^{\prime}}>\operatorname{dim} G_{a d}$ or equivalently that $\operatorname{dim} \gamma^{\prime}+l(w)>\operatorname{dim} \gamma+l(w)$ or that $\operatorname{dim} \gamma^{\prime}>\operatorname{dim} \gamma$. But from 0.1(a) we see that $\gamma$ is contained in the closure of $\gamma^{\prime}$; since $\gamma \neq \gamma^{\prime}$ it follows that $\operatorname{dim} \gamma^{\prime}>\operatorname{dim} \gamma$, as required.

Note that (a) and (b) provide, in the case where $C$ is elliptic, another characterization of $\Phi(C)$ which does not rely on the partial order on $\underline{\underline{G}}$.

\section{The MAin RESUlts}

1.1. In this section we assume that $p=2$. Let $V$ be a $\mathbf{k}$-vector space of finite dimension $\mathbf{n}=2 n \geq 4$ with a fixed nondegenerate symplectic form $():, V \times V \rightarrow \mathbf{k}$ and a fixed quadratic form $Q: V \rightarrow \mathbf{k}$ such that (i) or (ii) below holds:

(i) $Q=0$;

(ii) $Q \neq 0,(x, y)=Q(x+y)-Q(x)-Q(y)$ for $x, y \in V$.

Let $I s(V)$ be the group consisting of all $g \in G L(V)$ such that $(g x, g y)=(x, y)$ for all $x, y \in V$ and $Q(g x)=Q(x)$ for all $x \in V$ (a closed subgroup of $G L(V)$ ). Let $G$ be the identity component of $I s(V)$. Let $\mathcal{F}$ be the set of all sequences $V_{*}=\left(0=V_{0} \subset V_{1} \subset V_{2} \subset \cdots \subset V_{\mathbf{n}}=V\right)$ of subspaces of $V$ such that $\operatorname{dim} V_{i}=i$ for $i \in[0, \mathbf{n}],\left.Q\right|_{V_{i}}=0$ and $V_{i}^{\perp}=V_{\mathbf{n}-i}$ for all $i \in[0, n]$. Here, for any subspace $V^{\prime}$ of $V$ we set $V^{\prime \perp}=\left\{x \in V ;\left(x, V^{\prime}\right)=0\right\}$.

1.2. Let $p_{1} \geq p_{2} \geq \cdots \geq p_{\sigma}$ be a sequence in $\mathbf{Z}_{>0}$ such that $p_{1}+p_{2}+\cdots+p_{\sigma}=n$. (In the case where $Q \neq 0$ we assume that $\sigma$ is even.) For any $r \in[1, \sigma]$ we set $p_{\leq r}=\sum_{r^{\prime} \in[1, r]} p_{r^{\prime}}, p_{<r}=\sum_{r^{\prime} \in[1, r-1]} p_{r^{\prime}}$. We fix $\left(V_{*}, V_{*}^{\prime}\right) \in \mathcal{F} \times \mathcal{F}$ such that for any $r \in[1, \sigma]$ we have

(a) $\operatorname{dim}\left(V_{p_{<r}+i}^{\prime} \cap V_{p_{<r}+i}\right)=p_{<r}+i-r, \quad \operatorname{dim}\left(V_{p_{<r}+i}^{\prime} \cap V_{p_{<r}+i+1}\right)=p_{<r}+i-r+1$ if $i \in\left[1, p_{r}-1\right]$;

(b) $\operatorname{dim}\left(V_{p_{\leq r}}^{\prime} \cap V_{\mathbf{n}-p_{<r}-1}\right)=p_{\leq r}-r, \quad \operatorname{dim}\left(V_{p_{\leq r}}^{\prime} \cap V_{\mathbf{n}-p_{<r}}\right)=p_{\leq r}-r+1$. (Such $\left(V_{*}, V_{*}^{\prime}\right)$ exists and is unique up to conjugation by $I s(V)$.)

Let $B$ (resp. $B^{\prime}$ ) be the stabilizer in $G$ of $V_{*}$ (resp. $\left.V_{*}^{\prime}\right)$. Let $w$ be the relative position of the Borel subgroups $B, B^{\prime}$ (an element of the Weyl group of $G$ ) and let $C$ be the conjugacy class of $w$ in the Weyl group (it is an elliptic conjugacy class). 
A unipotent class $\gamma$ in $G$ is said to be adapted to $\left(V_{*}, V_{*}^{\prime}\right)$ if for some $g \in \gamma$ we have $g V_{i}=V_{i}^{\prime}$ for all $i$. Note that $\gamma$ is adapted to $\left(V_{*}, V_{*}^{\prime}\right)$ if and only if $C \dashv \gamma$.

There is a unique unipotent conjugacy class $\gamma$ in $G$ such that $\gamma$ is adapted to $\left(V_{*}, V_{*}^{\prime}\right)$ and some/any element of $\gamma$ has Jordan blocks of sizes $2 p_{1}, 2 p_{2}, \ldots, 2 p_{\sigma}$. (The existence of $\gamma$ is proved in [L1, 2.6, 2.12]; the uniqueness follows from the proof of [L1, 4.6].)

Theorem 1.3. Let $\gamma^{\prime}$ be a unipotent conjugacy class in $G$ which is adapted to $\left(V_{*}, V_{*}^{\prime}\right)$. Then $\gamma$ is contained in the closure of $\gamma^{\prime}$ in $G$.

The proof is given in $1.5-1.8$ (when $Q=0$ ) and in 1.9 (when $Q \neq 0$ ).

1.4. Let $\mathcal{T}$ be the set of sequences $c_{*}=\left(c_{1} \geq c_{2} \geq c_{3} \geq \ldots\right)$ in $\mathbf{N}$ such that $c_{m}=0$ for $m \gg 0$ and $c_{1}+c_{2}+\cdots=\mathbf{n}$. For $c_{*} \in \mathcal{T}$ we define $c_{*}^{*}=\left(c_{1}^{*} \geq c_{2}^{*} \geq c_{3}^{*} \geq \ldots\right) \in \mathcal{T}$ by $c_{i}^{*}=\left|\left\{j \geq 1 ; c_{j} \geq i\right\}\right|$ and we set $\mu_{i}\left(c_{*}\right)=\left|\left\{j \geq 1 ; c_{j}=i\right\}\right|(i \geq 1)$; thus we have

(a) $\mu_{i}\left(c_{*}\right)=c_{i}^{*}-c_{i+1}^{*}$.

For $i, j \geq 1$ we have

(b) $i \leq c_{j}$ iff $j \leq c_{i}^{*}$.

For $c_{*} \in \mathcal{T}$ and $i \geq 1$ we have

(c) $\sum_{j \in\left[1, c_{i}^{*}\right]}\left(c_{j}-i\right)+\sum_{j \in[1, i]} c_{j}^{*}=\mathbf{n}$.

Indeed, the left-hand side is

$$
\begin{aligned}
& \sum_{j \geq 1 ; i \leq c_{j}}\left(c_{j}-i\right)+\sum_{j \in[1, i], k \geq 1 ; c_{k} \geq j} 1=\sum_{j \geq 1 ; i \leq c_{j}}\left(c_{j}-i\right)+\sum_{k \geq 1} \min \left(i, c_{k}\right) \\
& =\sum_{j \geq 1 ; i \leq c_{j}}\left(c_{j}-i\right)+\sum_{k \geq 1 ; i \leq c_{k}} i+\sum_{k \geq 1 ; i>c_{k}} c_{k} \\
& =\sum_{j \geq 1 ; i \leq c_{j}} c_{j}+\sum_{k \geq 1 ; i>c_{k}} c_{k}=\sum_{j \geq 1} c_{j}=\mathbf{n} .
\end{aligned}
$$

For $c_{*}, c_{*}^{\prime} \in \mathcal{T}$ and $i \geq 1$ we have:

(d) $\sum_{j \in[1, i]} c_{j}^{*}=\sum_{j \in[1, i]} c_{j}^{\prime *}$ iff $\sum_{j \in\left[1, c_{i}^{*}\right]}\left(c_{j}-i\right)=\sum_{j \in\left[1, c_{i}^{\prime *}\right]}\left(c_{j}^{\prime}-i\right)$ and we have $\sum_{j \in[1, i]} c_{j}^{*} \geq \sum_{j \in[1, i]} c_{j}^{\prime *}$ iff $\sum_{j \in\left[1, c_{i}^{*}\right]}\left(c_{j}-i\right) \leq \sum_{j \in\left[1, c_{i}^{\prime *}\right]}\left(c_{j}^{\prime}-i\right)$.

This follows from (c) and the analogous equality for $c_{*}^{\prime}$.

For $c_{*}, c_{*}^{\prime} \in \mathcal{T}$ we say that $c_{*} \leq c_{*}^{\prime}$ if the following (equivalent) conditions are satisfied:

(i) $\sum_{j \in[1, i]} c_{j} \leq \sum_{j \in[1, i]} c_{j}^{\prime}$ for any $i \geq 1$;

(ii) $\sum_{j \in[1, i]} c_{j}^{*} \geq \sum_{j \in[1, i]} c_{j}^{*}$ for any $i \geq 1$.

We show the following:

(e) Let $c_{*}, c_{*}^{\prime} \in \mathcal{T}$ and $i \geq 1$ be such that $c_{*} \leq c_{*}^{\prime}, \sum_{j \in[1, i]} c_{j}^{*}=\sum_{j \in[1, i]} c_{j}^{*}$. Then $c_{i}^{*} \leq c_{i}^{*}$. If, in addition, we have $\mu_{i}\left(c_{*}\right)>0$, then $\mu_{i}\left(c_{*}^{\prime}\right)>0$.

We set $m=c_{i}^{*}, m^{\prime}=c_{i}^{*}$. From $c_{*} \leq c_{*}^{\prime}$ we deduce $\sum_{j \in[1, i-1]} c_{j}^{*} \geq \sum_{j \in[1, i-1]} c_{j}^{\prime *}$ (if $i=1$ both sums are zero); using the equality $\sum_{j \in[1, i]} c_{j}^{*}=\sum_{j \in[1, i]} c_{j}^{* *}$ we deduce $c_{i}^{*} \leq c_{i}^{\prime *}$; that is, $m \leq m^{\prime}$. From (d) we have $\sum_{j \in[1, m]}\left(c_{j}-i\right)=\sum_{j \in\left[1, m^{\prime}\right]}\left(c_{j}^{\prime}-i\right)$.

Hence

$$
\begin{aligned}
& \sum_{j \in[1, m]} c_{j}=\sum_{j \in\left[1, m^{\prime}\right]} c_{j}^{\prime}+\left(m-m^{\prime}\right) i \\
& \quad=\sum_{j \in[1, m]} c_{j}^{\prime}+\sum_{j \in\left[m+1, m^{\prime}\right]}\left(c_{j}^{\prime}-i\right) \geq \sum_{j \in[1, m]} c_{j}^{\prime} \geq \sum_{j \in[1, m]} c_{j} ;
\end{aligned}
$$


thus we have used $c_{*} \leq c_{*}^{\prime}$ and that for $j \in\left[m+1, m^{\prime}\right]$ we have $i \leq c_{j}^{\prime}$ (since $j \leq c_{i}^{\prime *}$, see (b)). It follows that the inequalities in (f) are equalities, hence $c_{j}^{\prime}=i$ for $j \in\left[m+1, m^{\prime}\right]$. Thus $\mu_{i}\left(c_{*}^{\prime}\right) \geq m-m^{\prime}$. This completes the proof of (e) in the case where $m>m^{\prime}$. Now assume that $m=m^{\prime}$. From $c_{*} \leq c_{*}^{\prime}$ we have $\sum_{j \in[1, m-1]} c_{j} \leq \sum_{j \in[1, m-1]} c_{j}^{\prime}$. Using this and (d) we see that

$$
\sum_{j \in[1, m]}\left(c_{j}-i\right)=\sum_{j \in[1, m]}\left(c_{j}^{\prime}-i\right) \geq \sum_{j \in[1, m-1]}\left(c_{j}-i\right)+c_{m}^{\prime}-i,
$$

hence $c_{m}-i \geq c_{m}^{\prime}-i$. From $\mu_{i}\left(c_{*}\right)>0$ and $c_{i}^{*}=m$ we deduce that $c_{m}=i$. (Indeed by 1.4(b) we have $i \leq c_{m}$; if $i<c_{m}$, then $i+1 \leq c_{m}$ and by 1.4(b) we have $m \leq c_{i+1}^{*} \leq c_{i}^{*}=m$, hence $c_{i+1}^{*}=c_{i}^{*}$ and $\mu_{i}\left(c_{*}\right)=0$, a contradiction.) Hence $c_{m}^{\prime} \leq i$. Since $c_{i}^{\prime *}=m$ we have also $i \leq c_{m}^{\prime}$ (see (b)), hence $c_{m}^{\prime}=i$. Thus $\mu_{i}\left(c_{*}^{\prime}\right)>0$. This completes the proof of (e).

1.5. In this subsection (and until the end of 1.8) we assume that $Q=0$. In this case we write $S p(V)$ instead of $I s(V)=G$. Let $u$ be a unipotent element of $S p(V)$. We associate to $u$ the sequence $c_{*} \in \mathcal{T}$ whose nonzero terms are the size of the Jordan blocks of $u$. We must have $\mu_{i}\left(c_{*}\right)=$ even for any odd $i$. We also associate to $u$ a map $\epsilon_{u}:\left\{i \in 2 \mathbf{N} ; i \neq 0, \mu_{i}\left(c_{*}\right)>0\right\} \rightarrow\{0,1\}$ as follows: $\epsilon_{u}(i)=0$ if $\left((u-1)^{i-1}(x), x\right)=0$ for all $x \in \operatorname{ker}(u-1)^{i}: V \rightarrow V$ and $\epsilon_{u}(i)=1$ otherwise; we have automatically $\epsilon_{u}(i)=1$ if $\mu_{i}\left(c_{*}\right)$ is odd. Now $u \mapsto\left(c_{*}, \epsilon_{u}\right)$ defines a bijection between the set of conjugacy classes of unipotent elements in $S p(V)$ and the set $\mathfrak{S}$ consisting of all pairs $\left(c_{*}, \epsilon\right)$ where $c_{*} \in \mathcal{T}$ is such that $\mu_{i}\left(c_{*}\right)=$ even for any odd $i$ and $\epsilon:\left\{i \in 2 \mathbf{N} ; i \neq 0, \mu_{i}\left(c_{*}\right)>0\right\} \rightarrow\{0,1\}$ is a function such that $\epsilon(i)=1$ if $\mu_{i}\left(c_{*}\right)$ is odd. (See [S, I,2.6]). We denote by $\gamma_{c_{*}, \epsilon}$ the unipotent class corresponding to $\left(c_{*}, \epsilon\right) \in \mathfrak{S}$. For $\left(c_{*}, \epsilon\right) \in \mathfrak{S}$ it will be convenient to extend $\epsilon$ to a function $\mathbf{Z}_{>0} \rightarrow\{-1,0,1\}$ (denoted again by $\epsilon$ ) by setting $\epsilon(i)=-1$ if $i$ is odd or $\mu_{i}\left(c_{*}\right)=0$.

Now let $\gamma, \gamma^{\prime}$ be as in 1.3. We write $\gamma=\gamma_{c_{*}, \epsilon}, \gamma^{\prime}=\gamma_{c_{*}^{\prime}, \epsilon^{\prime}}$ with $\left(c_{*}, \epsilon\right),\left(c_{*}^{\prime}, \epsilon^{\prime}\right) \in \mathfrak{S}$. Let $g \in \gamma_{c_{*}^{\prime}, \epsilon^{\prime}}$ be such that $g V_{*}=V_{*}^{\prime}$ and let $N=g-1: V \rightarrow V$. To prove that $\gamma$ is contained in the closure of $\gamma^{\prime}$ in $G$ it is enough to show that:

(a) $c_{*} \leq c_{*}^{\prime}$ and that for any $i \geq 1$, (b) and (c) below hold:

(b) $\sum_{j \in[1, i]} c_{j}^{*}-\max (\epsilon(i), 0) \geq \sum_{j \in[1, i]} c_{j}^{* *}-\max \left(\epsilon^{\prime}(i), 0\right)$;

(c) if $\sum_{j \in[1, i]} c_{j}^{*}=\sum_{j \in[1, i]} c_{j}^{\prime *}$ and $c_{i+1}^{*}-c_{i+1}^{\prime *}$ is odd, then $\epsilon^{\prime}(i) \neq 0$.

(See [S, II,8.2].) From the definition we see that $c_{i}=2 p_{i}$ for $i \in[1, \sigma], c_{i}=0$ for $i>\sigma$ and from [L1, 4.6] we see that $\epsilon(i)=1$ for all $i \in\{2,4,6, \ldots\}$ such that $\mu_{i}\left(c_{*}\right)>0$.

Now (a) follows from [L1, 3.5(a)]. Indeed, in loc.cit., it is shown that for any $i \geq 1$ we have $\operatorname{dim} N^{i} V \geq \Lambda_{i}$ where

$$
\Lambda_{i}=\sum_{j \geq 1 ; i \leq c_{j}}\left(c_{j}-i\right)=\sum_{j \in\left[1, c_{i}^{*}\right]}\left(c_{j}-i\right) .
$$

We have $\operatorname{dim} N^{i} V=\sum_{j \geq 1 ; i \leq c_{j}^{\prime}}\left(c_{j}^{\prime}-i\right)=\sum_{j \in\left[1, c_{i}^{\prime *}\right]}\left(c_{j}^{\prime}-i\right)$, hence by $1.4(\mathrm{~d})$ the inequality $\operatorname{dim} N^{i} V \geq \Lambda_{i}$ is the same as the inequality $\sum_{j \in[1, i]} c_{j}^{*} \geq \sum_{j \in[1, i]} c_{j}^{\prime *}$.

Note also that, by $1.4(\mathrm{~d})$,

(d) we have $\sum_{j \in[1, i]} c_{j}^{*}=\sum_{j \in[1, i]} c_{j}^{\prime *}$ iff $\operatorname{dim} N^{i} V=\Lambda_{i}$. 
1.6. Let $i \geq 1$. We show that:

(a) If $\mu_{i}\left(c_{*}\right)>0$ and $\sum_{j \in[1, i]} c_{j}^{*}=\sum_{j \in[1, i]} c_{j}^{\prime *}$, then $\epsilon^{\prime}(i)=1$.

By 1.4(e) we have $\mu_{i}\left(c_{*}^{\prime}\right)>0$. Since $\mu_{i}\left(c_{*}\right)>0$ we see that $i=2 p_{d}$ for some $d \in[1, \sigma]$. If $\mu_{i}\left(c_{*}^{\prime}\right)$ is odd, then $\epsilon^{\prime}(i)=1$ (by definition, since $i$ is even). Thus we may assume that $\mu_{i}\left(c_{*}^{\prime}\right) \in\{2,4,6, \ldots\}$. From our assumption we have that $\operatorname{dim} N^{i} V=\Lambda_{i}($ see $1.5(\mathrm{~d}))$.

Let $v_{1}, v_{2}, \ldots, v_{\sigma}$ be vectors in $V$ attached to $V_{*}, V_{*}^{\prime}, g$ as in [L1, 3.3]. For $r \in[1, \sigma]$ let $W_{r}, W_{r}^{\prime}$ be as in [L1, 3.4]; we set $W_{0}=0, W_{0}^{\prime}=V$. From [L1, 3.5(b)] we see that $N^{i} W_{d-1}^{\prime}=0$ at least if $d \geq 2$; but the same clearly holds if $d=1$. We have $v_{d} \in W_{d-1}^{\prime}$, hence $N^{2 p_{d}} v_{d}=0$ and

$$
\begin{aligned}
\left(N^{2 p_{d}-1}\left(v_{d}\right), v_{d}\right) & =\left(N^{p_{d}} v_{d}, N^{p_{d}-1} v_{d}\right)=\left((g-1)^{p_{d}} v_{d},(g-1)^{p_{d}-1} v_{d}\right) \\
& =\left(g^{p_{d}} v_{d}, v_{d}\right)=1 .
\end{aligned}
$$

(We have used that $\left(v_{d}, g^{k} v_{d}\right)=0$ for $k \in\left[-p_{d}+1, p_{d}-1\right]$ and $\left(v_{d}, g^{p_{d}} v_{d}\right)=1$; see [L1, 3.3(iii)].) Thus $\epsilon^{\prime}(i)=1$. This proves (a).

1.7. We prove $1.5(\mathrm{~b})$. It is enough to show that, if $\epsilon(i)=1$ and $\epsilon^{\prime}(i) \leq 0$, then $\sum_{j \in[1, i]} c_{j}^{*} \geq \sum_{j \in[1, i]} c_{j}^{\prime *}+1$. Assume this is not so. Then using 1.5(a) we have $\sum_{j \in[1, i]} c_{j}^{*}=\sum_{j \in[1, i]} c_{j}^{*}$. Since $\epsilon(i)=1$ we have $\mu_{i}\left(c_{*}\right)>0$; using $1.6($ a) we see that $\epsilon^{\prime}(i)=1$, a contradiction. Thus 1.5(b) holds.

1.8. We prove $1.5(\mathrm{c})$. If $i$ is odd, then $\epsilon^{\prime}(i)=-1$, as required. Thus we may assume that $i$ is even. Using 1.5(a) and 1.4(e) we see that $c_{i}^{*} \leq c_{i}^{\prime *}$.

Assume first that $c_{i}^{*}=c_{i}^{\prime *}$. From $\mu_{i}\left(c_{*}\right)=c_{i}^{*}-c_{i+1}^{*}, \mu_{i}\left(c_{*}^{\prime}\right)=c_{i}^{\prime *}-c_{i+1}^{\prime *}$ we deduce that $\mu_{i}\left(c_{*}\right)-\mu_{i}\left(c_{*}^{\prime}\right)=c_{i+1}^{* *}-c_{i+1}^{*}$ is odd. If $\mu_{i}\left(c_{*}^{\prime}\right)$ is odd, we have $\epsilon^{\prime}(i)=1$ (since $i$ is even); thus we have $\epsilon^{\prime}(i) \neq 0$, as required. If $\mu_{i}\left(c_{*}^{\prime}\right)=0$, we have $\epsilon^{\prime}(i)=-1$; thus we have $\epsilon^{\prime}(i) \neq 0$, as required. If $\mu_{i}\left(c_{*}^{\prime}\right) \in\{2,4,6, \ldots\}$, then $\mu_{i}\left(c_{*}\right)$ is odd so that $\mu_{i}\left(c_{*}\right)>0$ and then $1.6\left(\right.$ a) shows that $\epsilon^{\prime}(i)=1$; thus we have $\epsilon^{\prime}(i) \neq 0$, as required.

Assume next that $c_{i}^{*}<c_{i}^{\prime *}$. By $1.5\left(\right.$ a) we have $\sum_{j \in[1, i+1]} c_{j}^{*} \geq \sum_{j \in[1, i+1]} c_{j}^{\prime *}$; using the assumption of $1.5(\mathrm{c})$ we deduce that $c_{i+1}^{*} \geq c_{i+1}^{*}$. Combining this with $c_{i}^{*}<c_{i}^{*}$ we deduce $c_{i}^{*}-c_{i+1}^{*}<c_{i}^{*}-c_{i+1}^{*}$; that is, $\mu_{i}\left(c_{*}\right)<\mu_{i}\left(c_{*}^{\prime}\right)$. It follows that $\mu_{i}\left(c_{*}^{\prime}\right)>0$. If $\mu_{i}\left(c_{*}\right)>0$, then by $1.6\left(\right.$ a) we have $\epsilon^{\prime}(i)=1$; thus we have $\epsilon^{\prime}(i) \neq 0$, as required. Thus we can assume that $\mu_{i}\left(c_{*}\right)=0$. We then have $c_{i}^{*}=c_{i+1}^{*}$ and we set $\delta=c_{i}^{*}=c_{i+1}^{*}$. As we have seen earlier, we have $c_{i+1}^{*} \geq c_{i+1}^{\prime *}$; using this and the assumption of $1.5(\mathrm{c})$ we see that $c_{i+1}^{*}-c^{\prime *}{ }_{i+1}=2 a+1$ where $a \in \mathbf{N}$. It follows that $c^{\prime *}{ }_{i+1}^{*}=\delta-(2 a+1)$. In particular, we have $\delta \geq 2 a+1>0$.

If $k \in[0,2 a]$, we have $c_{\delta-k}^{\prime}=i$. (Indeed, assume that $i+1 \leq c_{\delta-k}^{\prime}$; then by 1.4(b) we have $\delta-k \leq c_{i+1}^{*}=\delta-(2 a+1)$ hence $k \geq 2 a+1$, a contradiction. Thus $c_{\delta-k}^{\prime} \leq i$. On the other hand, $\delta=c_{i}^{*}<c_{i}^{*}$ implies by $1.4(\mathrm{~b})$ that $i \leq c_{\delta}^{\prime}$. Thus $c_{\delta-k}^{\prime} \leq i \leq c_{\delta}^{\prime} \leq c_{\delta-k}^{\prime}$, hence $c_{\delta-k}^{\prime}=i$.)

Using 1.4(b) and $c_{i+1}^{*}=\delta-(2 a+1)$ we see that $c_{\delta-(2 a+1)}^{\prime} \geq i+1$ (assuming that $\delta-(2 a+1)>0)$. Thus the sequence $c_{1}^{\prime}, c_{2}^{\prime}, \ldots, c_{\delta}^{\prime}$ contains exactly $2 a+1$ terms equal to $i$, namely $c_{\delta-2 a}^{\prime}, \ldots, c_{\delta-1}^{\prime}, c_{\delta}^{\prime}$.

We have $i>c_{\delta+1}$. (If $i \leq c_{\delta+1}$, then from $1.4(\mathrm{~b})$ we would get $\delta+1 \leq c_{i}^{*}=\delta$, a contradiction.)

Since $\delta>0$, from $c_{i}^{*}=\delta$ we deduce that $i \leq c_{\delta}$ (see $1.4(\mathrm{~b})$ ); since $\mu_{i}\left(c_{*}\right)=0$ we have $c_{\delta} \neq i$ hence $c_{\delta}>i$. From the assumption of $1.5(\mathrm{c})$ we see that $\operatorname{dim} N^{i} V=\Lambda_{i}$ 
(see $1.5(\mathrm{~d})$ ). Using this and $c_{\delta}>i>c_{\delta+1}$ we see that [L1, 3.5] is applicable and gives that $V=W_{\delta} \oplus W_{\delta}^{\perp}$ and $W_{\delta}, W_{\delta}^{\perp}$ are $g$-stable; moreover, $g: W_{\delta} \rightarrow W_{\delta}$ has exactly $\delta$ Jordan blocks and each one has size $\geq i$ and $g: W_{\delta}^{\perp} \rightarrow W_{\delta}^{\perp}$ has only Jordan blocks of size $\leq i$. Since the $\delta$ largest numbers in the sequence $c_{1}^{\prime}, c_{2}^{\prime}, \ldots$ are $c_{1}^{\prime}, c_{2}^{\prime}, \ldots, c_{\delta}^{\prime}$ we see that the sizes of the Jordan blocks of $g: W_{\delta} \rightarrow W_{\delta}$ are $c_{1}^{\prime}, c_{2}^{\prime}, \ldots, c_{\delta}^{\prime}$. Since the last sequence contains an odd number $(=2 a+1)$ of terms equal to $i$ we see that $\epsilon_{\left.g\right|_{W_{\delta}}}(i)=1$. (Note that $($,$) is a nondegenerate symplectic$ form on $W_{\delta}$, hence $\epsilon_{\left.g\right|_{W_{\delta}}}(i)$ is defined as in 1.5.) Hence there exists $z \in W_{\delta}$ such that $N^{i} z=0$ and $\left(z, N^{i-1} z\right)=1$. This implies that $\epsilon_{g}(i)=1$; that is, $\epsilon^{\prime}(i)=1$. This completes the proof of 1.5(c) and also completes the proof of Theorem 1.3 when $Q=0$.

1.9. In this subsection we assume that $Q \neq 0$. Let $\gamma, \gamma^{\prime}$ be as in 1.3. We denote by $\gamma_{1}, \gamma_{1}^{\prime}$ the $I s(V)$-conjugacy class containing $\gamma, \gamma^{\prime}$, respectively; let $\gamma_{2}, \gamma_{2}^{\prime}$ be the $S p(V)$-conjugacy class containing $\gamma_{1}, \gamma_{1}^{\prime}$, respectively. Note that Theorem 1.3 is applicable to $\gamma_{2}, \gamma_{2}^{\prime}$ instead of $\gamma, \gamma^{\prime}$ and with $G$ replaced by the larger group $S p(V)$. Thus we have that $\gamma_{2}$ is contained in the closure of $\gamma_{2}^{\prime}$ in $S p(V)$ and then, using [S, II,8.2], we see that $\gamma_{1}$ is contained in the closure of $\gamma_{1}^{\prime}$ in $I s(V)$. We have $\gamma_{1}=\gamma$ (see [S, I,2.6]). If $\gamma_{1}^{\prime}=\gamma^{\prime}$, it follows that $\gamma$ is contained in the closure of $\gamma^{\prime}$ in $G$, as required. If $\gamma_{1}^{\prime} \neq \gamma^{\prime}$, then $\gamma_{1}^{\prime}=\gamma^{\prime} \sqcup \gamma^{\prime \prime}$ where $\gamma^{\prime \prime}=r \gamma^{\prime} r^{-1}(r$ is a fixed element in $I s(V)-G)$. We see that either $\gamma$ is contained in the closure of $\gamma^{\prime}$ or in the closure of $r \gamma^{\prime} r^{-1}$. In the last case we have that $r^{-1} \gamma r$ is contained in the closure of $\gamma^{\prime}$. But $r^{-1} \gamma r=\gamma$ so that in any case $\gamma$ is contained in the closure of $\gamma^{\prime}$. This completes the proof of Theorem 1.3 when $Q \neq 0$.

\section{REFERENCES}

[L1] G. Lusztig, From conjugacy classes in the Weyl group to unipotent classes, Represent. Theory 15 (2011), 494-530. MR2833465

[L2] G. Lusztig, Elliptic elements in a Weyl group: a homogeneity property, Represent. Theory 16 (2012), 189-211.

[S] N. Spaltenstein, Classes unipotentes et sous-groupes de Borel, Lecture Notes in Math., vol. 946, Springer-Verlag, 1982. MR672610 (84a:14024)

Department of Mathematics, Massachusetts Institute of Technology, Cambridge, Massachusetts 02139

Department of Mathematics, Northwestern University, Evanston, Illinois 60208 